\title{
New ICI Self Cancellation Scheme for OFDM Systems
}

\author{
S. E. D. Habib, Reem I. Sayed, Hisham M. Hamed, and Magdi Fikri
}

\begin{abstract}
Orthogonal Frequency Division Multiplexing (OFDM) is sensitive to Carrier Frequency Offset (CFO), which in turn causes Intercarrier Interference (ICI) and degrades system performance. During the last decade, several ICI self cancellation schemes were proposed in the literature. This paper introduces a new ICI self-cancellation scheme with an improved immunity against CFO errors as evidenced by Carrier to Interference Ratio (CIR) and Bit Error Rate (BER) analyses.
\end{abstract}

Index Terms-Carrier frequency offset (CFO), self cancellation (SC), intercarrier interference (ICI), CIR (carrier to interference ratio), OFDM (orthogonal frequency-division multiplexing).

\section{INTRODUCTION}

OFDM is an established modulation scheme in current broadband wireless mobile communication systems due to its high spectral efficiency and robustness to multi-path interference [1], [2]. A well-known disadvantage of the OFDM system is its sensitivity to frequency offset caused by oscillator inaccuracies and the Doppler shift in the channel. The carrier frequency offset causes loss of orthogonality, in which case the signals transmitted on each carrier are not independent of each other, thus leading to ICI Several techniques were developed to reduce the effect of frequency offset. These techniques can be grouped into two broad categories [3]. The first category is termed the frequency offset estimation and correction category [4], [5]. The second group is termed the frequency-offset reduction category, and is exemplified by references [6]-[13]. In this second category, either a guard interval or redundant modulation is necessary in order to achieve a trade-off between frequency offset sensitivity and bandwidth efficiency. In this paper, a new scheme belonging to the second category, the redundant modulation ICI selfcancellation schemes, is proposed that gives better performance compared to the existing ICI self-cancellation schemes.

This paper is organized as follows: Section II outlines briefly the ICI effect in OFDM systems. Section III reviews the published ICI self-cancellation techniques. Our ICI selfcancellation scheme is introduced in Section IV. Section V details a comparative CIR and BER performance study between the proposed scheme and earlier schemes. The conclusions of this paper are summarized in Section VI.

Manuscript received October 11, 2013; revised January 22, 2014

S. E. D. Habib, Reem I. Sayed, and Magdi Fikri are with Electronics and Electrical Communications Engineering Department, Cairo University, Cairo, Egypt (e-mail: serag@eng.cu.edu.eg; engreem@yahoo.com; magdi.fikri@gmail.com).

Hisham M. Hamed is with Electronics Department, National Telecommunications Institute, Cairo, Egypt (e-mail: h.hamed@varkonsemi.com).

\section{OFDM SYSTEMS AND ICI EFFECT}

In the OFDM systems, the transmitted signal in the time domain can be expressed as

$$
x(n)=\frac{1}{N} \sum_{l=0}^{N-1} X(l) e^{j 2 \pi \ln / N}
$$

where $x(n)$ denotes the $n^{\text {th }}$ sample of the OFDM transmitted signal, $X(l)$ denotes the modulated symbol within the $l^{\text {th }}$ subcarrier, and $\mathrm{N}$ is the number of the total subcarriers. Assuming an AWGN channel, the received signal in time domain can be written as

$$
y(n)=x(n) e^{\frac{j 2 \pi n \varepsilon}{N}}+w(n)
$$

where $\varepsilon$ is the normalized frequency offset, and is given by $\Delta f \mathrm{NT}_{\mathrm{s}} . \quad \Delta f$ is the frequency difference between the transmitted and received carrier frequencies and $T_{s}$ is the subcarrier symbol period. The term $w(n)$ is the AWGN introduced by the channel.

The effect of this frequency offset on the received symbol stream can be understood by considering the received symbol $Y(k)$ on the $k^{\text {th }}$ sub-carrier [14].

$$
\begin{aligned}
& Y(k)=X(k) S(0)+\sum_{l=0, l \neq k .}^{N-1} X(l)[S(l-k)]+n_{k} \\
& k=0,1, \ldots . N-1
\end{aligned}
$$

where $X(k)$ is the transmitted symbol (M-ary phase-shift keying (M-PSK), for example) for the $k^{\text {th }}$ subcarrier, $n_{k}$ is the $k^{\text {th }}$ component of the FFT of $w(n)$, and $S(l-k)$ are the complex coefficients for the ICI components in the received signal. The ICI components are the interfering signals transmitted on sub-carriers other than the $k^{\text {th }}$ sub-carrier. The complex coefficient $S(l-k)$ is given by[14].

$$
S(l-k)=\frac{\sin (\pi(l+\varepsilon-k))}{N \sin (\pi(l+\varepsilon-k) / N)} e^{j \pi\left(1-\frac{1}{N}\right)(l+\varepsilon-k)}
$$

The CIR is the ratio of the signal power to the power in the interference components. It serves as a good indication of the signal quality at high signal to noise ratios. Assuming standard transmitted data with zero mean and statistically independent transmitted symbols on the different subcarriers, the CIR is given by

$$
C I R=\frac{\left|S_{0}\right|^{2}}{\sum_{l=0, . .}^{N-1}\left|S_{l}\right|^{2}}
$$

\section{REVIEW OF ICI SELF CANCELLATION SCHEMES}

Several researchers proposed different ICI suppression schemes. Among those ICI suppression schemes, much 
attention has been paid to the ICI self-cancellation schemes due to its simplicity. The principle of this technique is to map each transmitted symbol onto a group of $r(r \geq 2)$ subcarriers, as shown in Fig. 1 and the ICI generated within the group can be self-cancelled through the characteristics of ICI coefficients.

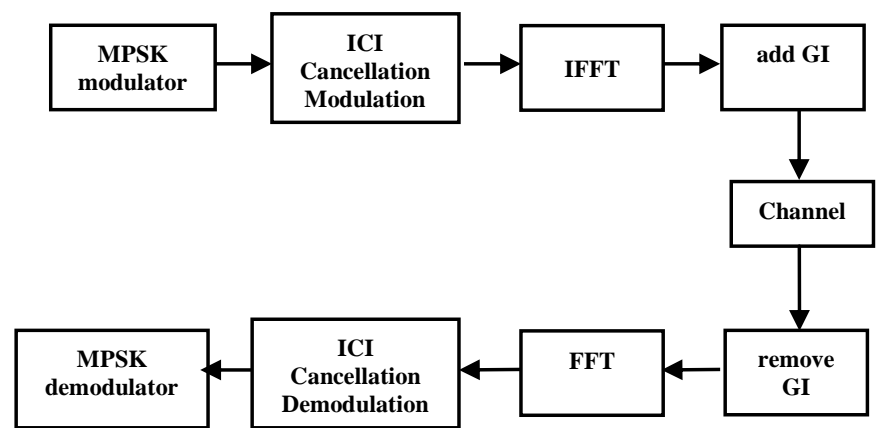

Fig. 1. The basic block diagram of an ICI Self Cancellation OFDM systems.

The first ICI self-cancellation technique was reported by Zhao [6], and is known as Adjacent Symbol Repetition (ASR). It depends on repeating the same symbol with opposite polarity on two adjacent subcarriers, $k$ and $(k+1)$, and then combining the received samples at the receiver as given by:

$$
\begin{aligned}
& Y^{\prime}(k)=Y(k)-Y(k+1) \\
& \quad=X(k)[-S(-1)+2 S(0)-S(1)]+ \\
& \sum_{l=0,2, \ldots l \neq k}^{N-1} X(l)[-S(l-k-1)+2 S(l-k)-S(l-k+1)]+n_{k}-n_{k+1}
\end{aligned}
$$

The Phase-Rotated Data Allocation (PRDA) scheme in [12] is closely related to ASR, where the same symbol is repeated with a phase shift $-\pi / 2$ on two adjacent subcarriers, $k$ and $(k+1)$. An improved form of PRDA was proposed in [13] where each symbol is repeated with different complex weights on four consecutive subcarriers, which degrades the throughput severely.

In Symmetric Symbol Repetition (SSR) scheme [7], the repeated symbols with opposite polarity are transmitted on symmetric subcarriers $k$ and $(N-k-1)$. Therefore, the data block becomes: $\mathrm{x}=\left(X_{0}, X_{1}, \ldots, X_{N / 2+1},-X_{N / 2+1}, \ldots . .,-X_{1}\right.$, $X_{0}$ ).The receiver combines the received samples $Y_{k}$ and $Y_{N-k}$ ${ }_{I}$ and the decision variable is expressed as

$$
\begin{aligned}
& Y^{\prime}(k)=Y(k)-Y(N-k-1) \\
& \quad=X(k)[-S(N-1)+2 S(0)-S(1-N)]+ \\
& \sum_{l=0 . .}^{N / 2-1} X(l)[-S(N-l-k-1)+S(l-k)+S(k-l)-S(l+k+1-N)] \\
& +n_{k}-n_{N-1-k}
\end{aligned}
$$

While ASR and SSR schemes are effective in reducing the ICI term on the received signal, the received signal still experiences the same phase rotation as a conventional OFDM.

In another technique, known as Adjacent Conjugate Symbol Repetition (ACSR)[8], the symbol $X_{k}$ on subcarrier $k$ is conjugated and repeated on subcarrier $(k+1)$. Therefore, the data block will be $\mathrm{x}=\left(X_{0}, X^{*}, \ldots X_{N / 2+1}, X^{*}{ }_{N / 2+1}\right)$, where * denotes complex conjugate. The receiver combines the received samples $Y_{k}$ and $Y_{k+1}$ through the relation:

$$
\begin{aligned}
Y^{\prime}(k)= & Y(k)+Y^{*}(k+1) \\
= & X(k)\left[S(0)+S^{*}(0)\right]+\sum_{l=0,2, \ldots, l \neq k}^{N / 2-1} X(l)\left[S(l-k)+S^{*}(l-k)\right] \\
& +\sum_{l=0,2, \ldots}^{N / 2-1} X *(l)[S(l+1-k)+S *(l-1-k)]+n_{K}+n^{*}{ }_{K+1}
\end{aligned}
$$

In Symmetric Conjugate Symbol Repetition (SCSR) [8],the modulated symbol $X_{k}$ is mapped on subcarriers $k$ and $(N-k-1)$. Therefore, the data block becomes $x=\left(X_{0}, X_{1}, \ldots\right.$, $\left.X_{N / 2+1}, \ldots \ldots, X^{*}{ }_{N / 2+1}, X^{*}, X_{0}^{*}\right)$.

The receiver combines the received samples $Y_{k}$ and $Y_{N-1-k}$ by:

$$
\begin{aligned}
& Y^{\prime}(k)=Y(k)+Y^{*}(N-1-k) \\
& \quad=X(k)\left[S(0)+S^{*}(0)\right]+\sum_{l=0,1, l \neq k .}^{N / 2-1} X(l)\left[S(l-k)+S^{*}(-l+k)\right] \\
& \left.+\sum_{l=0,1, \ldots}^{N / 2-1} X *(l)\left[S(N-1-l-k)+S^{*}(l+k-N+1)\right]+n_{K}+n^{*}{ }_{N-K-1}\right)
\end{aligned}
$$

The two previous schemes (ACSR, SCSR) mitigate the phase error, however the average CIR gain decreases with increasing frequency offset.

Another form of repetition; $\left(X_{k}, X_{k+1}=-X_{k}^{*}\right)$, is also considered in the peak-to-average power ratio (PAPR) reduction scheme, where the receiver combines the received samples $Y_{k}$ and $Y_{k+1}$ [9] using:

$$
\begin{aligned}
Y^{\prime}(k)= & Y(k)-Y^{*}(k+1) \\
= & X(k)\left[S(0)+S^{*}(0)\right]+\sum_{l=0,2, \ldots, l \neq k}^{N / 2-1} X(l)\left[S(l-k)+S^{*}(l-k)\right] \\
& +\sum_{l=0,2, \ldots}^{N / 2-1} X^{*}(l)\left[-S(l+1-k)-S^{*}(l-1-k)\right]+n_{K}-n^{*}{ }_{K+1}
\end{aligned}
$$

The PAPR reduction technique is closely related to ACSR technique. Unlike other ICI self-cancellation algorithms employing data repetition [6] or conjugate data repetition [7] within the same OFDM symbol interval, the Conventional Conjugate Cancellation (CC) Scheme [10] transmits two independent paths such that the ICI weighting coefficient for one path is the conjugate of that for the other path. The CC scheme achieves high CIR values when the frequency offset is small. However, the CIR performance of the CC scheme declines more rapidly with increased offset than those of other ICI cancellation schemes. This property makes the CC scheme undesirable at high frequency offset situations. A generalized form of CC scheme was proposed in [11], where one of the two paths is shifted by an adaptable phase angle relative to the other. The estimation of this adaptable phase angle requires a feedback from receiver to transmitter, which adds significant complexity to this scheme.

\section{PROPOSED ICI SELF-CANCELLATION SCHEMES}

In this paper, we propose a new ICI self-cancellation technique. The main idea of this technique is first introduced using a simple single carrier system, then it is applied to multicarrier modulation systems.

\section{A. Conjugate Phase Additive Single Carrier Transmission}

The main idea of the proposed scheme is first introduced 
for a simple single carrier system with QPSK modulation. Assume also that the effect of channel AWGN can be neglected, thus leaving the system with CFO errors only. Each symbol is conjugated and repeated to build a data pattern in the transmitted stream. This data pattern will be utilized at the receiver to cancel the CFO errors. Thus, the transmitted stream takes the form $X_{1} X_{1}{ }^{*} X_{2} X_{2}{ }^{*} X_{3} X_{3}{ }^{*} \ldots$. Note that the throughput of the system is half that of a classical QPSK, and therefore, its performance should be compared to a classical BPSK system. At the receiver, each symbol suffers a CFO phase error $\varphi(t)=2 \pi \Delta f$ t. Consequently, the received symbols $R_{1 A}$ and $R_{1 B}$ corresponding to transmitted symbols $X_{1}$ and $X_{1}{ }^{*}$ are given by:

$$
R_{1 A}=r_{1} e^{j\left(\theta_{1}+\varphi\right)} \quad \& \quad R_{1 B}=r_{1} e^{-j\left(\theta_{1}-\varphi\right)}
$$

where $r_{1}$ and $\theta_{1}$ are the magnitude and the phase of the received symbol corresponding to transmitted symbol $X_{1}$. We can now eliminate the effect of CFO phase shift completely if we calculate the output of the receiver as

- Get $R_{1 B}$ *.

- Multiply $R_{1 \mathrm{~A}}$ multiplied by $R_{1 B}{ }^{*}$. Note that the offset phase angle $\varphi$ is eliminated in the product.

- Calculate the received signal as

$$
Y_{1}=r_{1} e^{j \theta_{1}}
$$

Although equation 12 indicates that the CFO error is completely eliminated, the multiplication process has several disadvantages. First, constellation points of the lower half of the complex plane are folded on those of the upper half; leaving only half the constellation points usable. Also, multiplication complicates the symbol detection process considerably. It adds also significant hardware complexity. We, therefore, modified the algorithm in equation 12 as follows:

- Calculate phases of $R_{1 A}$ and $R_{1 B}{ }^{*}$. Find the average these two phases $\theta_{a v}$.

- Calculate the received signal as

$$
Y_{1}=r_{1} e^{j \theta_{a v}}
$$

Our proposed ICI self cancellation scheme is, therefore, labelled as conjugate phase additive scheme. Note also that complete CFO elimninaion is valid for higher order modulation schemes, provided that AWGN effect is negligible and the system is a single carrier system. Partial cancellation of the CFO errors takes place if any of these two conditions is invalid

\section{B. Conjugate Phase Additive Multi Carrier Transmission}

In this section, we apply the proposed modulation scheme introduced in section (IV-A) into OFDM system and we propose the next two conjugate techniques.

\section{1) Adjacent conjugate phase additive OFDM (ACPA)}

At the transmitter, we map the modulated data symbol $X$ on two subcarriers as $X$ and $X^{*}$, then the received symbol on subcarrier $k$ becomes

$$
Y(k)=\sum_{l=0,2, . .}^{N-1} X(l) S(l-k)+X^{*}(l) S(l+1-k)
$$

Similarly, on subcarrier $k+1$ we get

$$
Y^{*}(k+1)=\sum_{l=0,2, \ldots}^{N-1} X(l) S^{*}(l-k)+X^{*}(l) S^{*}(l-1-k)
$$

The receiver combines the received samples $Y_{k}$ and $Y_{k+1}$ by multiplying (Adding phases in polar form) the adjacent subcarriers after conjugating $Y_{k+1}$ using the following relation:

$$
\begin{gathered}
Y^{\prime}(k)=Y(k) \cdot Y^{*}(k+1) \\
=X^{2}(k)\left[S(0) S^{*}(0)\right]+\sum_{l=2,4, l \neq k}^{N-1} X^{2}(l)\left[S(l-k) S^{*}(l-k)\right]+ \\
\sum_{l=0,2, l \neq k .}^{N-1} X(l) X^{*}(l)\left[S(l+1-k) S^{*}(l-k)+S(l-k) S^{*}(l-k-1)\right]+ \\
\sum_{l=0,2, \ldots, l \neq k}^{N-1} X^{2} *(l)\left[S(l-k-1) S^{*}(l-k-1)\right]+n_{k} n^{*}{ }_{k+1}
\end{gathered}
$$

The first term in the right-hand side of (15) represents the desired signal, and the other terms are the ICI components.

Using ICI coefficient given by (15), the CIR of ACPA scheme can be expressed as

$$
C I R=\frac{\left|S_{0} S_{0}^{*}\right|^{2}}{\sum_{l=2,4, \ldots}^{N-1}\left|S_{l} S^{*}{ }_{l}\right|^{2}+\sum_{l=0,2}^{N-1}\left|S_{l+1} S_{l-1}^{*}\right|^{2}+\sum_{l=0,2}^{N-1}\left|S_{l+1} S_{l}^{*}+S_{l} S_{l-1}^{*}\right|^{2}}
$$

Phase shift of each subcarrier received symbol changes according to the change of the frequency offset. If the phase shift is significant enough for a particular modulation scheme, then the original data may be corrupted. For the proposed self cancellation scheme the phase rotation of the desired signal $X(k)$ caused by the frequency offset is

$$
\theta=\left\{\frac{\operatorname{Im}\left(S_{0} S_{0}^{*}\right)}{\operatorname{Re}\left(S_{0} S_{0}^{*}\right)}\right\}
$$

2) Symmetric conjugate phase additive $\operatorname{OFDM}(S C P A)$

In this scheme, the modulation symbol $X$ is mapped on $k$ and $k^{*}$ is mapped on subcarriers $k$ and $(N-k-1)$, respectively. Therefore, the data block becomes $x=\left(X_{0}, X_{1}, \ldots\right.$, $\left.X_{N / 2+1}, \ldots \ldots, X^{*}{ }_{N / 2+1}, X^{*}, X^{*}{ }_{0}\right)$.

The receiver combines the received symbols $Y_{k}$ and $Y_{N-1-k}$ and the decision variable becomes

$$
\begin{gathered}
Y^{\prime}(k)=Y(k) . Y^{*}(N-1-k) \\
=X^{2}(k)[S(0) S *(0)]+\sum_{l=1,2, l \neq k}^{N / 2-1} X^{2}(l)\left[S(l-k) S^{*}(k-l)\right]+ \\
\sum_{l=0,1, l \neq k}^{N / 2-1} X^{2} *(l)[S(N-l-k-1) S *(l-N+k+1]+ \\
\sum_{l=0,1, l \neq k .}^{N / 2-1} \quad X(l) X^{*}(l)\left[S(N-l-1-k) S^{*}(l-N+k+1)\right. \\
\left.+n_{k} n^{*}(l-N+k+1)\right]
\end{gathered}
$$

The CIR of SCPA scheme can be expressed as

$$
C I R=\frac{\left|S_{0} S_{0}^{*}\right|^{2}}{\sum_{l=1,2, \ldots}^{N / 2-1}\left|S_{l} S_{-l}^{*}\right|^{2}+\sum_{l=0,1}^{N / 2-1}\left|S_{N-l-1} S_{-(N-l-1)}^{*}\right|^{2}+\sum_{l=0,1}^{N / 2-1}\left|S_{N-l-1} S_{-l}^{*}+S_{l} S_{-(N-l-1)}^{*}\right|^{2}}
$$

\section{Simulation Results ANd PeRformance EVAluation}

\section{A. Performance of the ACPA / SCPA Techniques}

For the sake of maintaining the same spectral efficiency, the modulation types compared are BPSK for standard 
OFDM and QPSK for the proposed one.

Fig. 2 shows the CIR performance of the proposed schemes in decibels as a function of the normalized frequency offset, where $N=1024$.

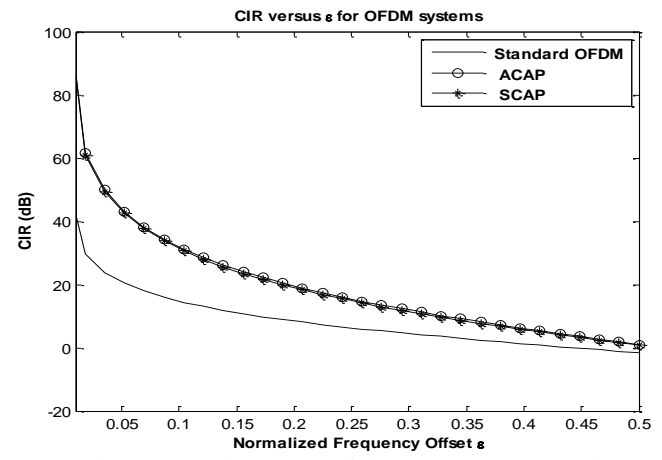

Fig. 2. The CIR performance of the ACPA, SCPA schemes.

From Fig. 2, we can see that both the proposed schemes are identical in CIR analysis and give about $40 \mathrm{~dB}$ to $10 \mathrm{~dB}$ CIR improvement for small to medium frequency offsets in the range $0<\varepsilon \leq 0.2$.

In terms of phase rotation error, Fig. 3 shows that the normal OFDM has a phase shift of about $45^{\circ}$ if the normalized frequency offset is 0.25 . This is a serious problem if the modulation scheme is chosen as QPSK or higher. Instead the proposed algorithms show a better performance and high immunity of ACPA/ SCPA algorithms to frequency offset errors.

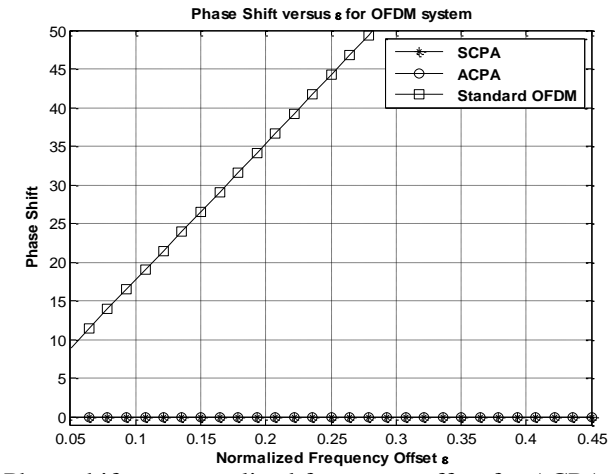

Fig. 3. Phase shift vs. normalized frequency offset for ACPA - SCPA schemes.

Also, the performance is evaluated in terms of BER versus $E_{b} / N_{0}$ ratio for different $\mathrm{CFO}$ values as shown in Fig. 4. Note that ACPA - Single Carrier is better than BPSK scheme in terms of AWGN noise and frequency offset elimination. ACPA BER sensitivity to $E_{b} / N_{\mathrm{o}}$, is approximately $1 \mathrm{~dB}$ higher than BPSK at $\varepsilon=0$.

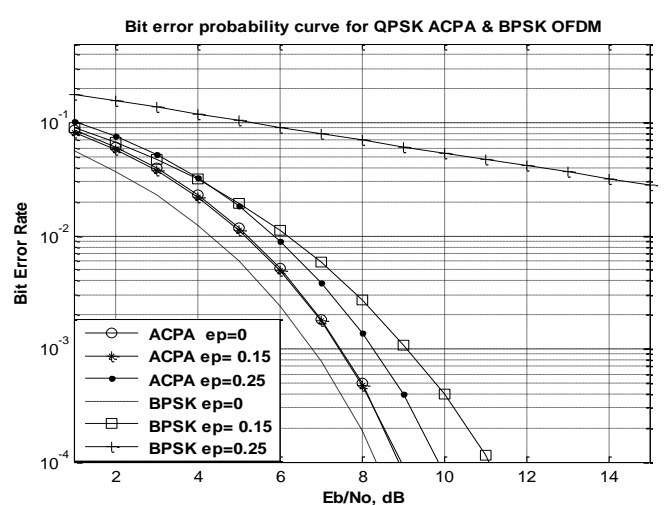

Fig. 4. BER versus $E_{b} N_{\mathrm{o}}$ for ACPA and BPSK Single carrier system.
For OFDM system, SCPA gives better performance than ACPA as illustrated by Fig. 5.

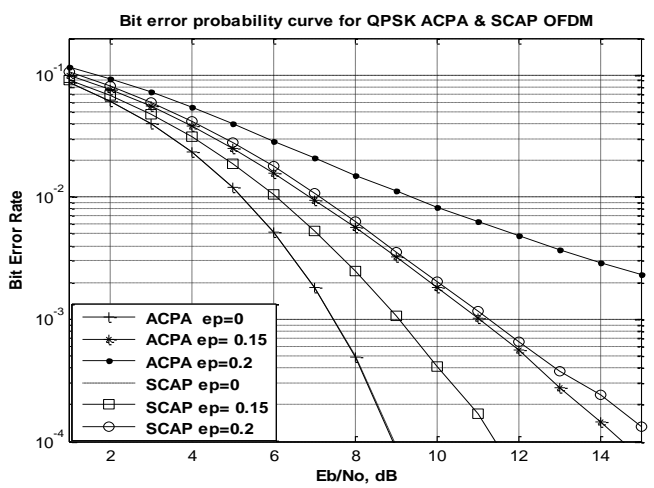

Fig. 5. BER versus $E_{b} N_{\mathrm{o}}$ of two proposed OFDM systems for different $\varepsilon$ values.

\section{B. Comparison with Other Schemes}

Now, we compare SCPA OFDM scheme; which gives better performance than ACPA scheme, with other schemes published in references [6]-[8], using CIR and phase rotation as a performance measure. It is clear from Fig. 6 that SCPA gives better CIR performance than standard and SCSR OFDM schemes. Although ASR, SSR schemes give better CIR performance than the proposed SCPA, they don't succeed in eliminating the phase rotation and completely cancel CFO. Also among all published techniques [6]-[13], the proposed technique gives the highest immunity to frequency offset variation.

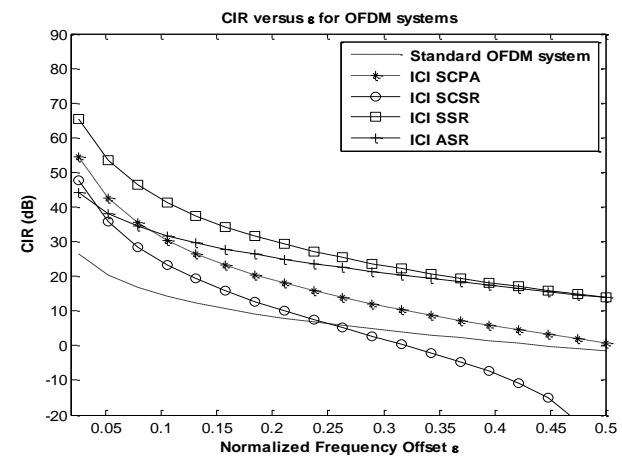

Fig. 6. CIR improvement using different ICI self-cancellation schemes.

The following paragraphs compare the BER performance of the proposed SCPA technique with ASR [6], and SCSR [8] schemes.

\section{1) SCPA QPSK scheme}

As illustrated in Fig. 7, for higher frequency offset, SCPA - QPSK scheme behaves better than both ASR for higher $\mathrm{E}_{\mathrm{b}} / \mathrm{N}_{\mathrm{o}}$ and SCSR for higher frequency offset values, $\varepsilon>0.1$.

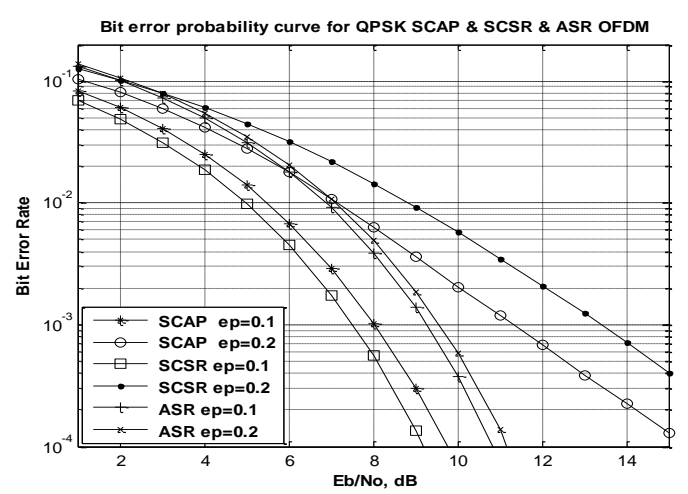

Fig. 7. BER comparison of SCPA and SCSR QPSK Scheme for $\varepsilon=0.1 \&$ 0.2 


\section{2) SCPA QAM16 scheme}

Fig. 8 shows the BER performance of SCAP, ASR and SCSR 16-QAM OFDM schemes for $N=64$ and with various frequency offset values with $\varepsilon=0.05 \& 0.15$.

SCPA outperforms the other schemes. It behaves better than ASR and offers an SNR gain of $3 \mathrm{~dB}$ over that of SCSR at $\varepsilon=0.15$ and BER of $10^{-2}$.

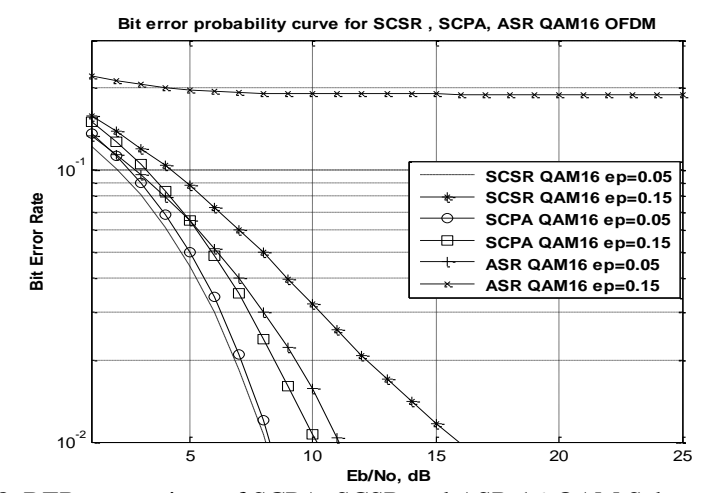

Fig. 8. BER comparison of SCPA,SCSR and ASR 16-QAM Scheme for $\varepsilon$ $=0.05 \& 0.15$.

\section{CONCLUSION AND FUTURE WORK}

In this paper, we have suggested two new ICI cancellation schemes to reduce the frequency offset sensitivity of the OFDM systems. These two systems are based on conjugate repetition of the symbols at the transmitter side and conjugate multiplication (adding the phases in polar form of complex numbers) of the data symbols at the output of the FFT block of the receiver. As opposed to previous ICI cancellation schemes, these two schemes combine very good CIR and BER performance even at high CFO errors.

\section{REFERENCES}

[1] S. Haykin, Communication Systems, 4th ed., Wiley, 2001.

[2] J. Proakis, Digital Communications, 5th ed., McGraw-Hill, 2007.

[3] R. Prasad, OFDM for Wireless Communications Systems, Artech House, London, UK, 2004.

[4] T. M. Schmidl and D. C. Cox, "Robust frequency and timing synchronization for OFDM," IEEE Trans. Commun., vol. 45, pp. 1613-1621, Dec. 1997.

[5] H. Liu and U. Tureli, "A high-efficiency carrier estimator for OFDM communications," IEEE Communications Letters, vol. 2, pp. 104-106, April 1998.

[6] Y. Zhao and S. G. Haggman, "Intercarrier interference selfcancellation scheme for OFDM mobile communication systems," IEEE Trans. Commun., vol. 49, no. 7, pp.1185-1191, July 2001.

[7] K. Sathananthan, R. M. A. P. Rajatheva, and S. B. Slimane, "Cancellation technique to reduce intercarrier interference in OFDM," IEE Elect. Lett., vol. 36, pp. 2078 -2079, Dec. 2000.

[8] K. Sathananthan, C. R. N. Athaudage, and B. Qin, "A Novel ICI cancellation scheme to reduce both frequency offset and IQ imbalance effects in OFDM," in Proc. Ninth International Symposium on Computers and Communications, July 2004, vol. 2, pp. 708-713.

[9] Y. Fu, S. G. Kang, and C. C. Ko, "A new scheme for PAPR reduction in OFDM systems with ICI self -cancellation," in Proc. Vehicular Technology Conference, 2002.

[10] V. K Dwivedi and G Singh, "An efficient BER analysis of OFDM systems with ICI conjugate cancellation method," in Proc. Progress In Electromagnetic Research Symposium, Cambridge, USA, July 2-6, 2008, pp. 166-171.

[11] C. L. Wang and Y. C. Huang, "Intercarrier interference cancellation using general phase rotated conjugate transmission for OFDM systems," IEEE Trans. on Comm., vol. 58, no. 3, pp. 812-819, Mar. 2010.

[12] C. Y. Yang, G. R. Lee, and J. H. Wen, "ICI self-cancellation scheme with phase-rotated data allocation in OFDM systems," in Proc.
International Conference on Advanced Information Technologies, April 2010, Taichung, Taiwan.

[13] M. Rahman, P. K. Dey, and M. F. R. U. Rashid, "Improved ICI self cancellation scheme for phase rotation error reduction in OFDM system," International Journal of Information and Electronics Engineering, vol. 2, no. 2, March 2012.

[14] N. Sreekanth and M. N. GiriPrasad, "Comparative BER analysis of mitigation of ICI through SC, ML and EKF methods In OFDM systems," International Journal of Electronics Communication and Computer Technology, vol. 2, issue 4, 2012.

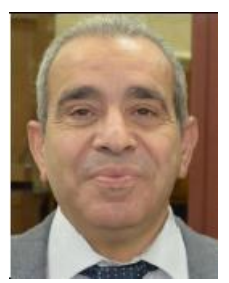

S. E. D. Habib was born in Egypt, 1947. He received the B.Sc. degree from Cairo University, Egypt in 1968; the M. Sc. Degree in solid state physics from the AUC, Egypt in 1971; and the M.Sc. and the Ph.D. degrees in electrical engineering from University of Toronto, Canada, in 1973 and 1978 respectively. He occupied several teaching and research positions at Cairo University, Egypt, University College of Swansea, Wales, U.K., Kuwait University, Kuwait, and Qatar University, Qatar. He was chairman of the Electrical Engineering Department, Qatar University for the academic year 1997/1998. He was the co-chairman of the International Conference on Microelectronics (ICM'03) held in Cairo in 2003. He is currently a professor emeritus at the Electronics and Communication Department, Faculty of Engineering, Cairo University. Dr. Habib's current research interests include VLSI design, SoC, MEMS, and semiconductor devices. He has authored and co-authored two books, 80 papers in refereed international journals and conferences, and one patent. In 1985, Dr. Habib was awarded the Egyptian State Encouragement Prize for his research in semiconductor devices. In 1995, he received the Egyptian Legion of Distinction (first class). He is a life member of IEEE.

Reem I. Sayed received the B.Sc. degree from Ain Shams University, Egypt in 2000. She received the M.Sc. from Cairo University, Egypt in 2004. She is currently a Ph.D. student in Cairo University, Egypt. Her current main research interests include communications systems, VLSI and embedded systems design. She is the embedded system design manager in VLSI Design Center, R\&D Department, Electronics Factory, AOI, Egypt.

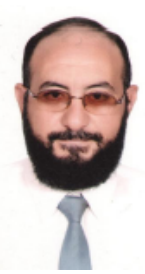

Hisham M. Hamed received the B.Sc with honors in 1989, the MSc. in 1994, and the PhD. in 2002, all in electronics and electronic communications, from the Faculty of Engineering at Cairo University. He was the VLSI department manager at the R\&D center, IEP, (Bahgat Group), from April 15th 2001 to Feb 2003, where he supervised the design of an MPEG decoder chip. He was appointed assistant professor of Electronics at the National Telecommunications Institute (NTI) in April 2003. From November 2005 to Feb. 2007 he was a consultant for SySDSoft, where he was involved in developing the baseband PHY, as well as the hardware design, for a BlueTooth 2.0 chip. He is currently the chief technical officer of Varkon semiconductors, which he co-founded in 2008. His research interests include hardware implementations of DSP algorithms for communications applications, and physical layer hardware design for wireless communications systems. Dr. Hamed is a member of the Center for Wireless Studies, at the Faculty of Engineering, Cairo University.

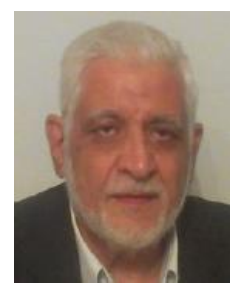

Magdi Fikri was born in Cairo, Egypt, 1942 and received his B. Sc. and M. Sc. degrees, both in electrical engineering from Cairo University, Cairo, Egypt in 1963 and 1970, respectively, and his Ph. D. in electrical engineering from the University of Bradford, Bradford, UK, in 1976. He joined the General Egyptian Aero Organization as an R \& D engineer from 1963 to 1971 , and the Department of Electronics and Electrical Communications Engineering, Cairo University, since March 1971 till now, where he is currently an emeritus professor. He was a research assistant with the Postgraduate School of Electrical Engineering, University of Bradford, England from 1973 to 1976, and a visiting scholar at the Ecole National Superieure des Telecommunications, Paris, in 1978. He was seconded as an associate professor to Qatar University, Electrical Engineering Department from 1982 to 1988, and as dean of Faculty of Computer Science and Information Systems, October 6 University from 1996 to 1998. His areas of interest include speech and image signal processing, wireless communication systems, signal processing for communications and software defined radio. 